\title{
Interactions between kaolinite clay and AOT
}

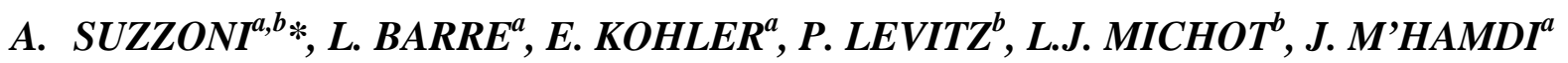 \\ ${ }^{a}$ IFP Energies nouvelles, 1 et 4 avenue du Bois-Préau, 92852, Rueil Malmaison, FRANCE \\ ${ }^{b}$ Laboratoire Physicochimie des Electrolytes et Nanosystèmes interfaciaux (PHENIX), UMR 8234, Université Pierre et Marie Curie, 75005 Paris, \\ FRANCE
}

\author{
A R T I C LE INF O \\ Article history: \\ Received 00 December 00 \\ Received in revised form 00 January 00 \\ Accepted 00 February 00

\section{Keywords:} \\ Kaolinite \\ Bis(2-ethylhexyl) \\ sulfosuccinate sodium \\ AOT \\ Adsorption \\ Stabilization \\ Orientation
}

\begin{abstract}
A B S T R A C T
Interactions between kaolinite (KGa-2) and a typical anionic surfactant bis(2-ethylhexyl) sulfosuccinate sodium (AOT) have been study to obtain a better knowledge of this system.

Firstly, adsorption isotherms of AOT on kaolinite are realized as a function of pH. Stability experiments have been performed between the surfactant and the clay mineral and the bed sediments structure have been studied with Small Angle X-ray Scattering (SAXS).

In acidic media, the shape of the adsorption isotherm leads to the following suggestions: firstly, for surfactant concentration below the CMC a surfactant monolayer is adsorbed on the positively charged edge surfaces, and then, for surfactant concentrations above the CMC, a bilayer is adsorbed on the edge surfaces. Consequently, in the first case, the surface becomes hydrophobic and in the second case, the surface becomes hydrophilic and this assumption is verified with hydrophobicity tests. Under alkaline conditions no surfactant is adsorbed on surfaces confirming the adsorption on the edge surfaces in acidic conditions. In agreement, with previous studies [1,2] significant stabilization of kaolinite suspensions can be observed in the presence of high surfactant concentration, in both $\mathrm{pH}$ conditions. Finally, structural studies by SAXS provide data about the organization of particles in the bed sedimentation.
\end{abstract}

\section{Introduction}

Interactions between clay minerals and surfactants are of prime importance in numerous industrial applications such as for instance water treatment $[3,4]$, ceramics, drilling muds, paints or mineral flotation [5]. In most of these applications, the aim is to maximize the interactions between the mineral and the surfactant and therefore, as clay minerals are mostly negatively charged, numerous studies deal with cationic surfactants/clay interactions [69]. In some specific applications however, strong interactions between clay minerals and surfactants would be detrimental. This is the case of Enhanced Oil Recovery, where surfactants solutions are injected into oil reservoirs for increasing oil yield [10-12]. In that context, strong interactions between rock minerals and surfactants would lead to significant losses, and consequently anionic molecules are the most used.

In terms of clay minerals, various species are present in reservoir rocks, such as kaolinite, chlorite, illite, smectites... Kaolinite is a dioctahedral clay mineral, belonging to the TO family. Its crystal structure is then formed by the association of one octahedral layer $(\mathrm{O})$ and one tetrahedral layer $(\mathrm{T})$. Interactions between anionic surfactant and kaolinite have been studied in the past [1315]. In particular, detailed analysis of the features of surfactant adsorption isotherms (shape and position) was performed to derive information about interaction mechanisms [16].

Still, to our knowledge, the effect of anionic surfactant adsorption on the structuration of kaolinites suspensions has rarely been studied in detail. In the present study, we will then analyze anionic surfactant adsorption on kaolinite and examine suspension structure along the adsorption isotherm. This will be achieved by combining adsorption isotherms measurements with (i) visual observations of the time evolution of the suspensions and (ii) small angle $\mathrm{X}$ ray scattering measurements of selected samples. Such analyses will be carried out in both acidic and alkaline conditions, as $\mathrm{pH}$ should play a major role. Indeed, in kaolinite, the basal faces are non-charged whereas edge surfaces bear broken bonds that generate $\mathrm{pH}$ dependent charges [17]. In acidic media, kaolinite edges will then be positively charged in contrast with alkaline conditions where edge surfaces will mainly be negative. 


\section{Material and Methods}

\subsection{Material}

\subsubsection{Clay mineral}

The kaolinite used in the present study is a natural clay mineral KGa-2, from Georgia, purchased from the Source Clays Minerals repository at the University of Missouri. The structural formula can be written as $\left(\mathrm{Al}_{3.66} \mathrm{Fe}^{3+}{ }_{0.07} \mathrm{Ti}_{0.16}\right)$ $\left(\mathrm{Si}_{4.00}\right) \mathrm{O}_{10}(\mathrm{OH})_{8}$ and the cation exchange capacity is 3.3 $\mathrm{meq} / 100 \mathrm{~g}$ (data from Clay Minerals Society website). Clay suspensions were purified and homoionized with sodium following a procedure used for swelling clays [18-20]. A $45 \mathrm{~g} / \mathrm{L}$ clay suspension was first exchanged three times in $1 \mathrm{M} \mathrm{NaCl}$ for 24 hours and centrifuged between each exchange. The suspension was then washed by dialysis with deionized water until the conductivity was below 5 $\mu \mathrm{S} / \mathrm{cm}$. After recovery from the dialysis tubes, the suspension was then transferred into Imhoff cones and sedimented for $24 \mathrm{~h}$. After this time, only the supernatant containing fine particles was recovered. Purity was checked by $\mathrm{X}$ ray diffraction and infrared spectroscopy. These analyses revealed only the presence of traces of anatase $(1.2 \% \mathrm{w})$ already mentioned in a previous study [21].

\subsubsection{Organic molecule}

The anionic surfactant used is bis(2-ethylhexyl) sulfosuccinate sodium (Aerosol-OT or AOT) purchased from Sigma Aldrich (BioXtra with a purity $\geq 99 \%$ ). Its chemical formula is $\mathrm{C}_{20} \mathrm{H}_{37} \mathrm{NaO}_{7} \mathrm{~S}$ and the molecular weight is $444.56 \mathrm{~g} / \mathrm{mol}$. The Critical Micellar Concentration (CMC) was determined with Wilhelmy plate method using $\mathrm{K} 100(\mathrm{C})$ tensiometer from Krüss with a platinum plate. The $\mathrm{CMC}$ is around $2.5 \mathrm{mM}$ for this surfactant in agreement with previous studies [22-26].

\subsection{Characterization techniques}

X-ray diffraction was carried out using a X'Pert PRO (PANalytical) diffractometer. The wavelength of the incident beam is: $\lambda=1.5418 \AA$. Preparations were either oriented preparations on a glass slide or disoriented preparation placed in glass capillaries.

Infrared spectra were obtained with IR-ATR (Infrared Attenuated Total Reflectance) diamond Vertex 70, from Bruker. The spectra were recorded between $4000 \mathrm{~cm}^{-1}$ and $400 \mathrm{~cm}^{-1}$. The powder was directly deposited on diamond crystal.

\subsection{Adsorption isotherm}

The depletion method was used to obtain adsorption isotherms that were all measured at room temperature. Surfactant solutions were prepared at different concentrations by successive dilutions.. $\mathrm{pH}$ was controlled by adding $\mathrm{NaOH}$ or $\mathrm{HCl}$. The part of the AOT phase diagram [27] considered is the micellar phase $\left(\mathrm{L}_{1}\right)$. Consequently, initial concentrations $\left(C_{i n i}\right)$ of surfactant varied between $0.1 \mathrm{CMC}$ and $2 \mathrm{CMC}$. These solutions were then mixed directly in centrifugation tubes with kaolinite suspensions at a fixed solid content and stirred Adsorption kinetics in such a system are rather fast, as adsorption equilibrium is reached within a few hours $[14,28]$. In the present case, adsorption times of 16 hours were chosen. The liquid phase was then separated from the solid one by centrifuging for 20 minutes at $10000 \mathrm{~g}$. The supernatant was then recovered, further filtered with a 0.2 $\mu \mathrm{m}$ syringe filter, to ensure that the liquid phase does not contain any fine particles. Finally, the surfactant concentration in the supernatant is measured with Hyamine 1622 titration, HPLC with ELSD detector (Evaporative Light Scattering Detector) in acidic media and with Total Organic Carbon under alkaline conditions. Preliminary blank experiments carried out in the absence of clay minerals showed that no surfactant was adsorbed during the experimental protocol, neither on filters nor on centrifugation tubes. The adsorbed quantity is calculated as the difference between initial and equilibrium concentrations, taking into account clay mass, solution volume, and clay specific surface area.

$$
Q_{a d s}=\frac{\left(C_{i n i}-C_{e q}\right) \cdot V_{s o l}}{m_{\text {clay }} \cdot S_{p}}
$$

With $C_{i n i}$ the initial surfactant concentration $[\mathrm{mol} / \mathrm{L}], C_{e q}$ the equilibrium concentration $[\mathrm{mol} / \mathrm{L}], V_{\text {sol }}$ the solution volume [L], $m_{\text {clay }}$ the clay mineral mass $[\mathrm{g}], S_{p}$ the specific surface area of the mineral $\left[\mathrm{m}^{2} / \mathrm{g}\right]$ and $Q_{a d s}$ expressed in $\left[\mathrm{mol} / \mathrm{m}^{2}\right]$.

Details about errors calculation for the adsorbed quantity are provided in Poirier's thesis [13] and are described in the appendix. In short, the error made on the adsorbed amount with the depletion method is minimized by the expression:

$$
\frac{\Delta Q_{a d s}}{Q_{a d s}} \leq \frac{x . C_{e q}}{C_{i n i}-C_{e q}}
$$

where $\mathrm{x}$ is the error relative to the experimental method used to determine equilibrium concentrations.

The error on the adsorbed amounts is higher when equilibrium concentration and initial concentration are close.

\subsection{Stability experiments}

The surfactant solutions used for determining adsorption isotherms were mixed with clay suspensions in vials to follow how the presence of anionic surfactant affects the stability of kaolinite particles. A clay suspension is first placed in each vial and surfactant solutions are then added. Each vial was mixed by hand-shaking to homogenize the sample. Then, one picture is taken every 30 seconds to observe the time evolution of sedimentation.

\subsection{Surface hydrophobicity experiments}

Cyclohexane was added to the samples containing kaolinite particles and surfactant solutions with various concentrations and after shaking the location of the solid particles was examined by visual inspection.

\subsection{SAXS experiments}


Small Angle X-ray Scattering experiments were used using a homemade device: the X-ray radiation coming from a rotating anode X-ray generator (Rigaku MM07) is reflected by a Xenocs $($ multilayer parabolic mirror to provide a monochromatic $(\lambda=1.54 \AA)$ parallel beam. Two pairs of low scattering crossed slits are used to define the beam size $\left(\approx 0.8 \times 0.8 \mathrm{~mm}^{2}\right.$ on the sample). The scattered intensity is measured using a $2 \mathrm{D}$ proportional detector (Rigaku). The wave vector $\mathrm{q}$, defined by $\mathrm{q}=4 \pi / \lambda \sin (\theta)$, where $2 \theta$ is the angle between the incident and scattered beams, has the dimension of a reciprocal length and can therefore be regarded as an inverse meter stick. Measurements have been conducted in a wave vector range of $3.10^{-2} \AA^{-1}$ up to $1 \AA^{-1}$. Three samples were prepared, in acidic media, kaolinite suspension was mixed with three solutions: one with no surfactant, one with a concentration below the $\mathrm{CMC}$ and one with a concentration above the CMC. Quartz capillaries have been filled with these preparations and flame sealed. SAXS acquisitions were made under vacuum and at different time of the particles sedimentation.

\section{Results and discussion}

\subsection{Particles characterization}

Particle sizes were determined by Scanning Transmission Electron Microscopy (STEM) using Nova nano Scanning Electron Microscope mode operating at $15 \mathrm{kV}$ in bright field STEM. One drop of a dilute suspension $(\sim 10 \mathrm{mg} / \mathrm{L})$ in $\mathrm{NaOH}$ solution at $\mathrm{pH} 10$ was deposited on a gold-coated copper grid and dried under infrared light. Around 400 particles were counted to determine the size properties of kaolinite. According to such an analysis, the average diameter is $230 \mathrm{~nm}$ and the particles are highly polydisperse (polydispersity $\approx 95 \%$ ). The first quartile is found to be $97 \mathrm{~nm}$ and the third quartile $268 \mathrm{~nm}$. These values correspond to other studies [29] except for the average diameter which is smaller than in the literature. This can be explained by the fact that counted particles were distinguished at the aggregate periphery while larger particles were hidden in the middle of aggregates. Consequently, the mean diameter could be slightly underestimated. Particles display polygonal shape and, for some particles clear hexagons could be differentiated, as shown on Figure 1. These anisotropic particles have an average aspect ratio around 10 [29], much less important than some other clay minerals, such as smectite for instance.

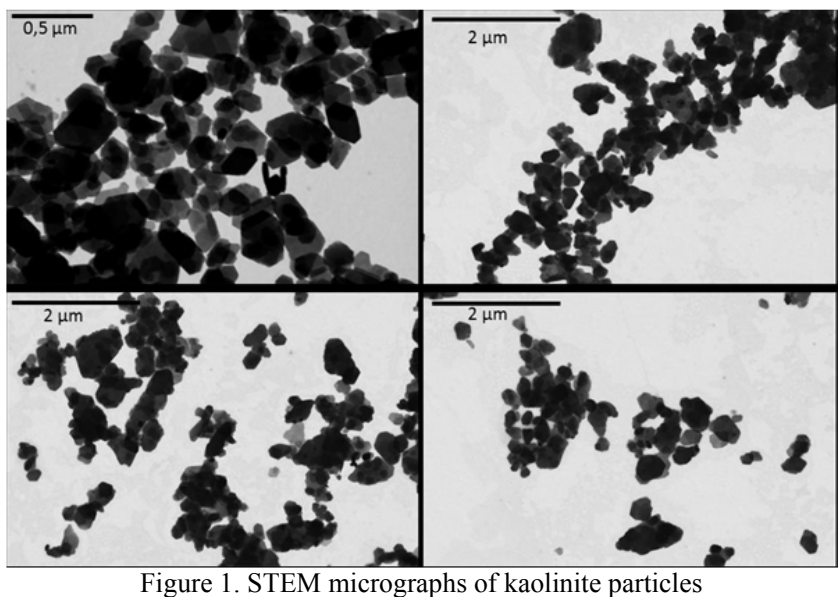

The specific surface area was determined using the adsorption of nitrogen at $77 \mathrm{~K}$ and analysis using the BET approach, was found to be $21 \mathrm{~m}^{2} / \mathrm{g}$, in agreement with the literature $[30,31]$.

The point of zero charge (PZC) corresponds to the $\mathrm{pH}$ value where the average superficial charge is zero. Potentiometric titration yielded a PZC value of 4 for KGa2 kaolinite [32].

\subsection{Adsorption isotherms}

AOT retention isotherms on kaolinite particles in both acidic and alkaline conditions are displayed on Figure 2. The representation of the experimental isotherm data with an $\mathrm{x}$ coordinate of $\mathrm{Ceq} / \mathrm{CMC}$ helps one extracting useful thermodynamic information [16]. The retention isotherms appear to be independent of the solid/liquid ratio, which shows that adsorption sensu stricto is the main retention mechanism. The $\mathrm{pH}$ of the suspension has a major effect on adsorption isotherms. Under alkaline conditions, the adsorbed amounts are close to zero. In such conditions kaolinite is negatively charged and therefore the interactions with anionic surfactant molecules are mainly repulsive. A significantly different situation is observed in acidic conditions where measurable adsorbed amounts are observed in the whole equilibrium concentration range.

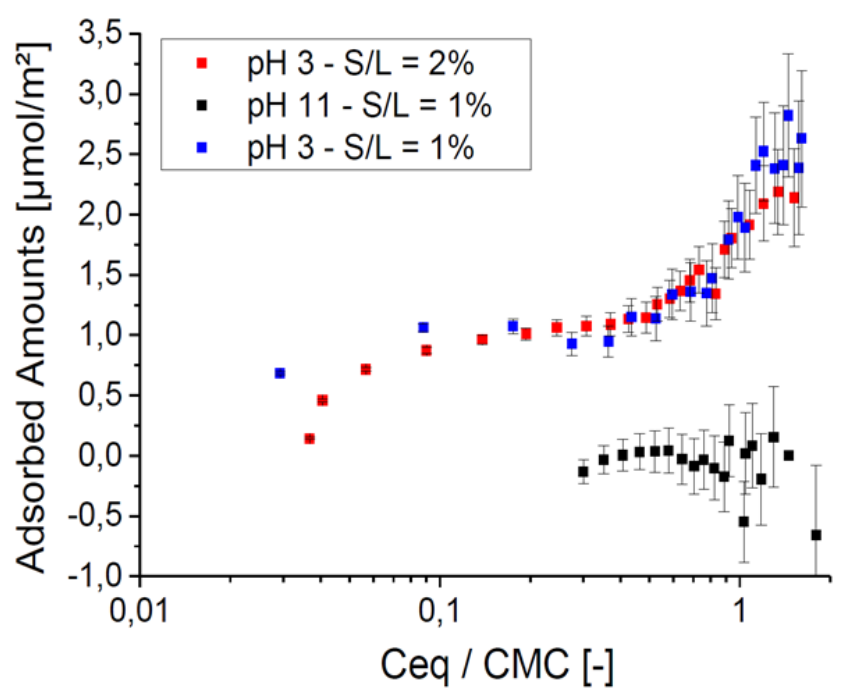

Figure 2. Influence of $\mathrm{pH}$ and clay concentration on adsorption isotherms of AOT on kaolinite particles

The isotherm displays two plateaus. On the second one, the adsorbed amount is around twice that obtained on the first one, and the corresponding equilibrium concentration is close to the $\mathrm{CMC}\left(\mathrm{C}_{\mathrm{eq}} / \mathrm{CMC} \approx 1\right)$. For a better interpretation of these isotherms it is relevant to try to estimate the equivalent surface area corresponding to this second plateau. This can be calculated according to the following equation

$$
S=N a \cdot \Gamma \cdot A_{A O T}
$$

where $N a$ is the Avogadro number $\left[\mathrm{mol}^{-1}\right], \Gamma$ the adsorbed amount $[\mathrm{mol} / \mathrm{g}], A_{A O T}$ the area occupied by one AOT molecule $\left[\mathrm{m}^{2}\right]$.

Using an area per AOT molecule of $57 \AA^{2}$ [33], the adsorbed amount at the second plateau yields an equivalent surface area of $14.4 \mathrm{~m}^{2} / \mathrm{g}$. Such a value is significantly 
lower than the total surface area of $\mathrm{KGa} 2$ kaolinite and significantly higher than the edge surface area whose value was estimated at $3.9 \mathrm{~m}^{2} / \mathrm{g}$ on the basis of high-resolution argon adsorption measurements [31]. Considering that at acidic $\mathrm{pH}$, the edge faces of kaolinite are positively charged, a possible interpretation of the shape of the adsorption isotherms could be to assign the first plateau to the completion of one monolayer on the edge faces, and the second one to the completion of a bilayer on the edge faces. The adsorbed amounts do not match perfectly with such an assumption, as they are slightly higher on both plateaus. Such a small discrepancy could be tentatively assigned to limited additional adsorption on basal faces mediated by the counter ion, a phenomenon already evidenced in other studies dealing with the adsorption of anionic surfactants on kaolinite [13]. According to such an interpretation, at the first plateau, as adsorption occurs through an electrostatic interaction between surface sites and the polar head of the surfactant molecule, the surface should be hydrophobic. In contrast, adsorption of the second layer could occur through interactions between adsorbed chains and the hydrophobic tails of AOT molecules, which would expose polar heads towards the solution, resulting in a hydrophilic surface. The relevance of such a situation can be tested by performing partition experiments between water and cyclohexane.

Results of these experiments are presented in Figure 3A. In the first vial (vial 1) in which no surfactant was contacted with kaolinite, all particles are located at the bottom, i.e. in the aqueous phase. The situation is markedly different is the second vial (vial 2) that corresponds to a sample located on the first plateau of the adsorption isotherm. Indeed, in that case, kaolinite particles are concentrated in the top phase, i.e. in cyclohexane, which reveals their hydrophobic character. Finally, in the third vial (vial 3) that corresponds to a sample located on the second plateau of the isotherm, most kaolinite particles are in the aqueous phase revealing the hydrophilic nature of the surfaces. As a control experiment, similar samples were prepared in alkaline conditions (Figure 3B). In that case, except for changes in sedimentation behavior and for the presence of air bubbles, kaolinite particles are always located in the aqueous phase. These macroscopic tests, though remaining

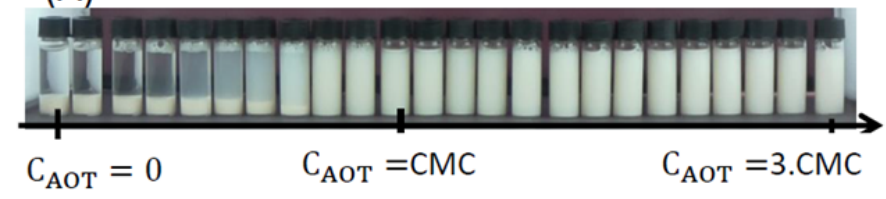

(A)

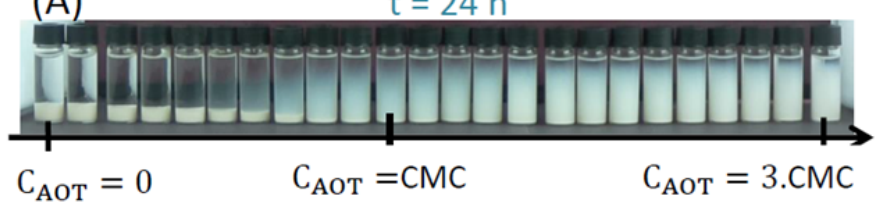

qualitative, tend to support the existence of a mono/bi layer of adsorbed surfactant on the first/second plateau of the isotherm, respectively.

Additional information can be derived from these simple tests. Indeed, in both acidic and alkaline conditions, surfactant addition induces changes in the sedimentation behavior of the samples that appear to be stabilized. It then seemed relevant to study such a behavior in greater details.

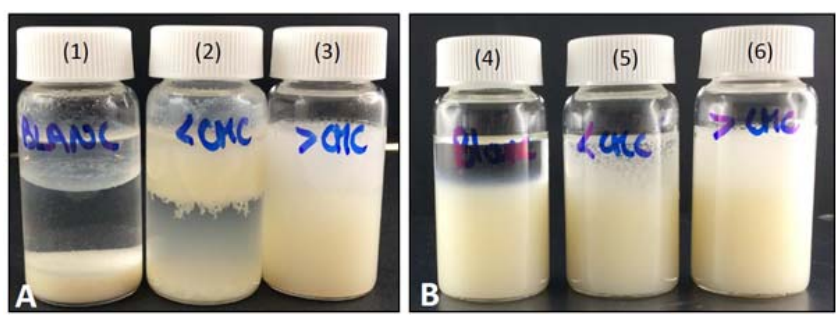

Figure 3. Partition experiments between water and cyclohexane in acidic media (A) and under alkaline conditions (B) after 10 minutes of settling. The cyclohexane phase is at the top and the aqueous phase is the inferior part. In vials (1) $\mathrm{C}_{\mathrm{AOT}}=0 . \mathrm{CMC}, \mathrm{pH} 3$; (2) $\mathrm{C}_{\mathrm{AOT}}=0.5 . \mathrm{CMC}, \mathrm{pH} 3$; (3) $\mathrm{C}_{\mathrm{AOT}}=3 . \mathrm{CMC}, \mathrm{pH} 3$; (4) $\mathrm{C}_{\mathrm{AOT}}=0 . \mathrm{CMC}, \mathrm{pH} 11 ;$ (5) $\mathrm{C}_{\mathrm{AOT}}=0.5 . \mathrm{CMC}, \mathrm{pH}$ $11 ;(6) \mathrm{C}_{\mathrm{AOT}}=3 . \mathrm{CMC}, \mathrm{pH} 11$

\subsection{Stabilization experiments}

Figure 4 presents optical pictures of kaolinite suspensions conditioned with increasing amounts of AOT in acidic (Figure 4A) and alkaline (Figure 4B) conditions obtained 30 minutes, 24 hours and a few months after their preparation.

In the absence of surfactant, solution $\mathrm{pH}$ influences the sedimentation behavior of kaolinite. Indeed, in acidic media, kaolinite particles settle after 20 minutes while, under alkaline conditions, particles are still in suspension after this time. After 24 hours, the sediment bed is higher in acidic media and such a difference is still observed after a few months settling. It then appears that changes in sedimentation speed are correlated to different particles organization in the sediments. Particles in acidic conditions then seem to be slightly more aggregated than in alkaline conditions, which may also explain differences in sediment organization, aggregation leading to less dense packings. Such a point may be assigned to differences in absolute (B)
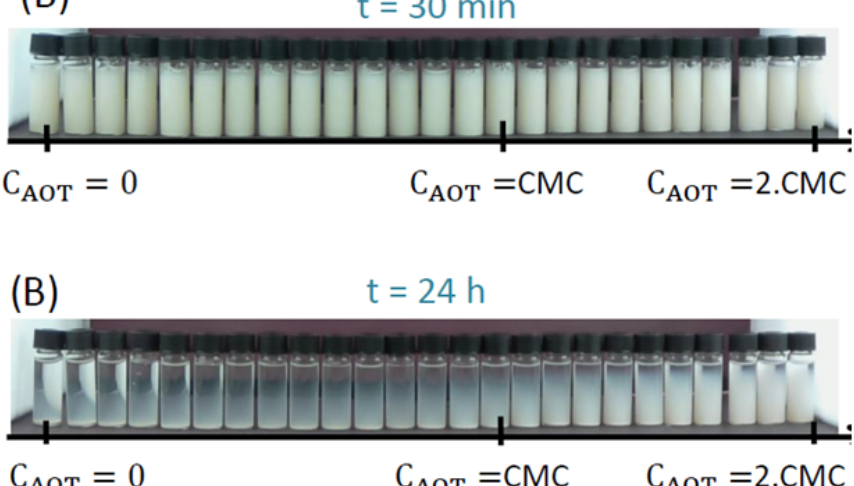

$\mathrm{C}_{\mathrm{AOT}}=\mathrm{CMC}$

$\mathrm{C}_{\mathrm{AOT}}=2 . \mathrm{CMC}$

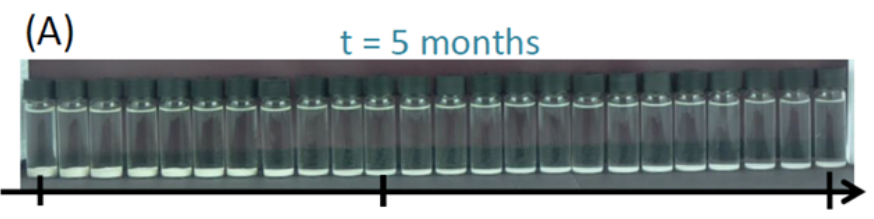

(B)

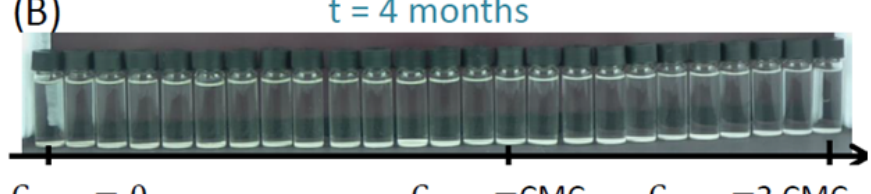

$\mathrm{C}_{\mathrm{AOT}}=0$

$\mathrm{C}_{\mathrm{AOT}}=\mathrm{CMC}$

$\mathrm{C}_{\mathrm{AOT}}=3 . \mathrm{CMC} \quad \mathrm{C}_{\mathrm{AOT}}=0$

$\mathrm{C}_{\mathrm{AOT}}=\mathrm{CMC}$

$\mathrm{C}_{\mathrm{AOT}}=2 . \mathrm{CMC}$

Figure 4. Stabilization experiments: in each tube from the left to the right the surfactant concentration increase : (A) under acidic conditions and (B) under alkaline conditions. At $\mathrm{pH} 3$, the surfactant concentration increases of $90 \mathrm{mg} / \mathrm{L}$ in each vial from the left to the right, except on five last vials the surfactant concentration increases of $160 \mathrm{mg} / \mathrm{L}$. 
values of the surface charge between acidic and alkaline conditions. Indeed, with a PZC around 4, the magnitude of the positive charge at $\mathrm{pH} 3$ is lower than that of the negative charge at $\mathrm{pH} 11$ as revealed by potentiometric titration experiments [21]. Still, this point clearly deserves further investigation and detailed studies of sediment structure could provide fruitful information on that topic.

For both $\mathrm{pH}$ conditions, surfactant addition clearly stabilizes the suspension and the effect is particularly striking for initial surfactant concentrations above the CMC. In such conditions, total sedimentation is achieved only after a few weeks. After a few months, in acidic conditions, the size of the sediment bed is significantly lower than for surfactant-free samples.

In acidic conditions, the influence of AOT can be linked to its adsorption on the edge faces. It can be foreseen that the presence of surfactants bilayers surrounding the particles contributes to electrostatic stabilization through increased repulsions and also may prevent aggregation Such an explanation does not apply to alkaline conditions as no surfactant adsorption occurs in these conditions. Depletion phenomena could be invoked to explain suspension stabilization, but direct evidence supporting such an interpretation is currently lacking.

\subsection{Structural studies Particles orientation}

In order to gather more information about the structure of the sediments obtained in various conditions at acidic $\mathrm{pH}$, SAXS experiments were carried out for different sedimentation times. The incoming $X$ Ray beam illuminates the sample placed in a capillary. The scattering intensity is recovered on a bidimensional detector. The principle of the experiment is represented on Figure 5.

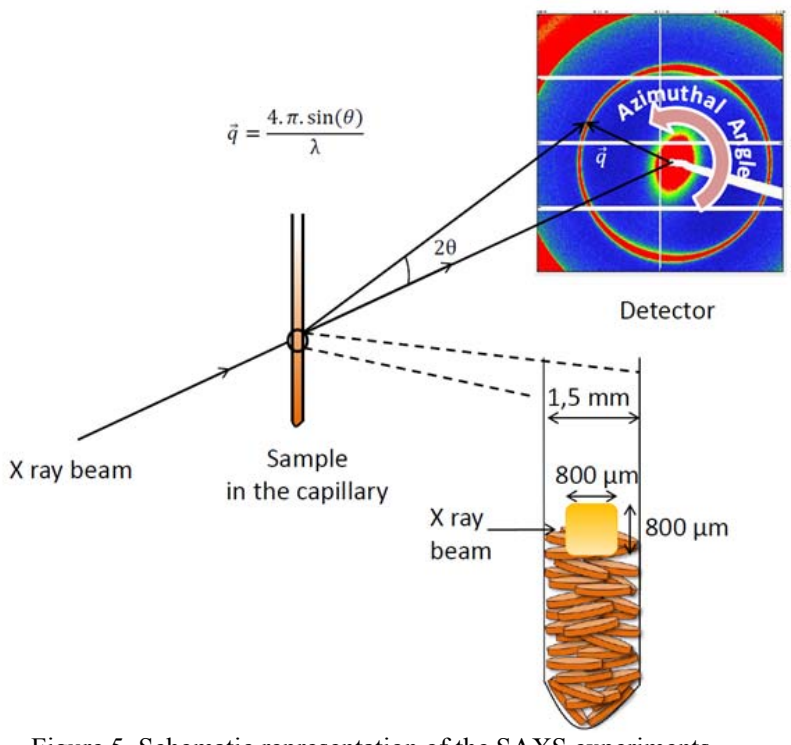

Figure 5. Schematic representation of the SAXS experiments.

Figure 6 displays SAXS pattern of three samples obtained for initial AOT concentrations of $0,0.5^{*} \mathrm{CMC}$ and $3 * \mathrm{CMC}$ after 13 days of sedimentation. The bidimensional pattern corresponding to the sample conditioned with the highest surfactant concentration is markedly distinct from the two other ones. Indeed, preferential orientation is revealed by this pattern both at low angle where an ellipsoidal shape of the scattering halo is observed and at higher angle where the intensity of the Bragg peak corresponding to the (001) plane of kaolinite at $\mathrm{q}=0.87 \AA^{-1}$ varies with the scattering angle.
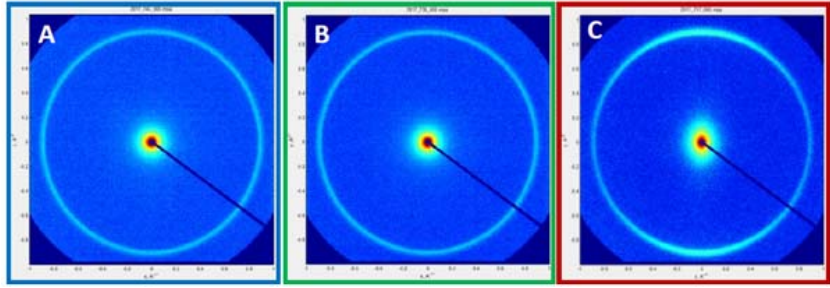

Figure 6 . The 2D SAXS pattern of sediment bed after 13 days of sedimentation (A) $\mathrm{C}_{\mathrm{AOT}}=0 . \mathrm{CMC}$; $\left.\mathrm{B}\right) \mathrm{C}_{\mathrm{AOT}}=0.5 . \mathrm{CMC}$; (C) $\mathrm{C}_{\mathrm{AOT}}=3 . \mathrm{CMC}$. Surfactant solutions were prepared at $\mathrm{pH} 3$.

Figure 7 displays the evolution of the intensity of this peak as a function of the azimuthal angle. For the sample with conditioned with $3 * \mathrm{CMC}$, a periodic evolution of the intensity can be observed whereas the two other samples reveal a constant intensity. The periodic evolution can be fitted using a Maier-Saupe [34-36] type orientation function according to :

$$
\left.f(\psi)=A+B \cdot \exp \left(m \cdot \cos ^{2}(\psi-\alpha) * \frac{\pi}{180}\right)\right)
$$

where $\mathrm{A}$ is a background term, B an intensity multiplicative term, $\mathrm{m}$ an orientation parameter, $\psi$ the azimuthal angle [degree ${ }^{\circ}$ ] and $\alpha$ related to the orientation angle [degree ${ }^{\circ}$.

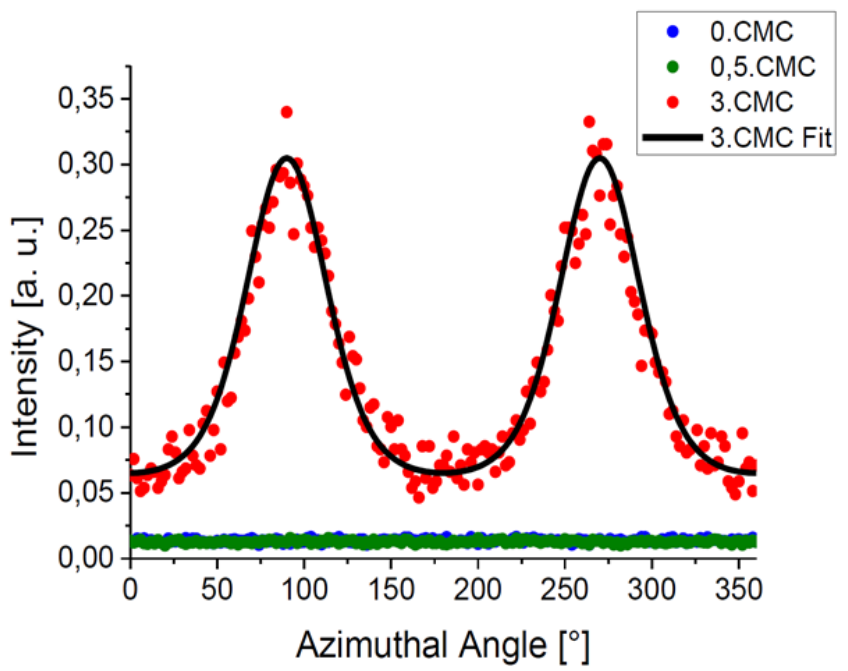

Figure 7. The intensity evolution of the first peak of Bragg versus azimuthal angle. The solid line is the fit used to determine an orientation parameter.

As revealed by these preliminary SAXS experiments, the presence of high surfactant amounts (> CMC) in acidic conditions clearly results in a preferred orientation of the particles in the sediment. Such enhanced orientation could be linked to either the slower sedimentation rate allowing better and easier particles reorganization in the sediment, or/and to lubrication phenomena in the sediment. Such information is important for a better understanding of fluids flows in clay porous media. The arrangement of particles in the porous media has indeed consequences on the permeability and thus, controls to some extent the migration of water and solutes [37].

A pictorial representation of the phenomena that could occur in the system is presented in Figure 8. In acidic conditions, AOT is adsorbed onto kaolinite surfaces and 
partition experiments reveal a change in wettability below the CMC. Then, particles are stabilized in suspension when AOT concentration increases. Furthermore, SAXS results reveal that, for high AOT amounts, particles are oriented in the sediment.

Under alkaline conditions, no surfactant is adsorbed on kaolinite surfaces but particles are still stabilized in suspension, essentially at medium to high AOT concentration. Additional experiments, particularly SAXS ones should be performed to precisely determine how particles are oriented in the sediment under both acidic and alkaline conditions. Still, sedimentation experiments point towards a better orientation of kaolinite particles in the sediment in alkaline conditions.

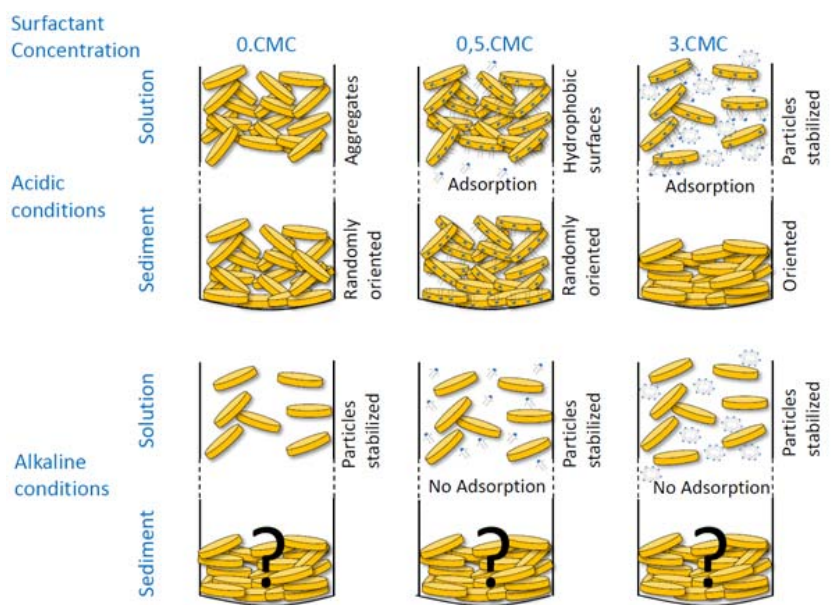

Figure 8. Synthesis scheme of possible interactions between kaolinite particles and AOT as a function of $\mathrm{pH}$ and surfactant concentration

To analyze the lubrication phenomena of the surfactant more experiments are needed. It would be particularly worthwhile to analyze samples with different solid/liquid ratios and to use microfocused X-ray beams, such as those available in synchrotron facilities, to follow the evolution of both concentration and orientation in the sediment as a function of height in the capillary. This will be the object of future publications.

\section{Acknowledgments}

We thank the anonymous reviewers for their careful reading of our study and their many constructive comments and suggestions.

\section{Appendix. Errors calculation}

Starting from the equation applying the rest method

$$
Q_{a d s}=\frac{\left(C_{\text {ini }}-C_{\text {é } q}\right) \cdot V_{\text {sol }}}{m_{\text {clay }} \cdot S_{p}}
$$

With $C_{i n i}$ the initial surfactant concentration $[\mathrm{mol} / \mathrm{L}], C_{e q}$ the equilibrium concentration $[\mathrm{mol} / \mathrm{L}], V_{\text {sol }}$ the solution volume [L], $m_{\text {clay }}$ mass [g], $S_{p}$ the specific surface area of the mineral $\left[\mathrm{m}^{2} / \mathrm{g}\right]$ and $Q_{a d s}$ expressed in $\left[\mathrm{mol} / \mathrm{m}^{2}\right]$, it comes:
$\frac{\Delta Q_{a d s}}{Q_{a d s}}=\frac{\Delta V}{V}+\frac{\Delta m}{m}+\frac{\Delta S}{S}+\frac{\Delta C_{i n i}}{C_{i n i}-C_{e q}}+\frac{\Delta C_{e q}}{C_{i n i}-C_{e q}}$

Considering that:

- The specific surface is constant from sample to sample $\rightarrow \Delta S=0$

- The error on the volume is constant and equal to $\frac{\Delta V}{V}=\frac{0.07}{10}=7.10^{-3}$

- The error on mass : $\frac{\Delta m}{m}=\frac{10^{-4}}{0.2}=5.10^{-4}$

- The error on the initial concentration if it is prepared by weighing and diluted in one liter: $\Delta C_{\text {ini }} \approx 10^{-4}$

$\frac{\Delta Q_{a d s}}{Q_{a d s}}=7.5 .10^{-3}+\frac{10^{-4}}{C_{i n i}-C_{e q}}+\frac{\Delta C_{e q}}{C_{i n i}-C_{e q}}$

The two first terms are insignificant with regard to the last one. Consequently, we can write :

$$
\begin{gathered}
\frac{\Delta Q_{a d s}}{Q_{a d s}} \geq \frac{\Delta C_{e q}}{C_{i n i}-C_{e q}} \\
\Delta C_{e q}=x \cdot C_{e q}
\end{gathered}
$$

With $\mathrm{x}$ is relative error of the experimental method to measure the equilibrium concentration.

$$
\frac{\Delta Q_{a d s}}{Q_{a d s}} \geq \frac{x \cdot C_{e q}}{C_{i n i}-C_{e q}}
$$

The error on the adsorbed amounts is higher when equilibrium concentration and initial concentration are close. 


\section{References}

[1] G. Lagaly, Principles of flow of kaolin and bentonite dispersions, Applied Clay Science 4 (1989) 105-123.

[2] J. Welzen, H.N. Stein, J.M. Stevels, C. Siskens, The influence of surface-active agents on kaolinite, Journal of colloid and interface science 81 (1981) 455-467.

[3] M.S. Zbik, R.S.C. Smart, G.E. Morris, Kaolinite flocculation structure, Journal of colloid and interface science 328 (2008) 73-80.

[4] M.S. Zbik, Y.-F. Song, R.L. Frost, Kaolinite flocculation induced by smectite addition - a transmission X-ray microscopic study, Journal of colloid and interface science 349 (2010) 86-92.

[5] D. Tao, X. Zhou, D. Kennedy, P. Dopico, J. Hines, Improved Phosphate Flotation Using Clay Binder, Separation Science and Technology 45 (2010) 604609.

[6] S. Xu and S. A. Boyd, Cationic Surfactant Adsorption by Swelling and Nonswelling Layer Silicates.

[7] S. Lee, S. Kim, Adsorption of naphthalene by HDTMA modified kaolinite and halloysite, Applied Clay Science 22 (2002) 55-63.

[8] M. Janek, G. LAGALY, Interaction of a cationic surfactant with bentonite: A colloid chemistry study, Colloid Polym Sci 281 (2003) 293-301.

[9] C. M. Ouellet-Plamondon, J. Stasiak, A. Al-Tabbaa, The effect of cationic, non-ionic and amphiphilic surfactants on the intercalation of bentonite, Colloids and Surfaces A: Physicochemical and Engineering Aspects 444 (2014) 330-337.

[10] Q. Liu, M. Dong, W. Zhou, M. Ayub, Y. Zhang, S. Huang, Improved oil recovery by adsorptiondesorption in chemical flooding, Journal of Petroleum Science and Engineering 43 (2004) 75-86.

[11] T. Amirianshoja, R. Junin, A. Kamal Idris, O. Rahmani, A comparative study of surfactant adsorption by clay minerals, Journal of Petroleum Science and Engineering 101 (2013) 21-27.

[12] S. Iglauer, Y. Wu, P. Shuler, Y. Tang, W. A. Goddard, New surfactant classes for enhanced oil recovery and their tertiary oil recovery potential, Journal of Petroleum Science and Engineering 71 (2010) 23-29.

[13] J.-E. Poirier, Etudes des mécanismes accompagnant l'adsorption des tensio-actifs ioniques sur les solides, dans le cas des systèmes à interactions faibles application à la récupération par voie chimique du pétrole contenu dans les gisements gréseux, Ecole Nationale Supérieure de Géologie Appliquée et de Prospection Minière. Institut national polytechnique de Lorraine. 1984.

[14] J.E. Poirier, J.M. Cases, Anionic surfactant adsorption onto silicate minerals the role of the cations, Colloids and Surfaces 55 (1991) 333-344.

[15] J. Rouquerol and S. Partyka, Adsorption of surfactants on rocks: Microcalorimetric approach applied to tertiary oil recovery, J. Chem. Tech. Biotechnol. 31 (1981) 584-592.

[16] J.M. Cases, F. Villieras, Thermodynamic model of ionic and nonionic surfactants adsorption-abstraction on heterogeneous surfaces, Langmuir 8 (1992) 12511264.
[17] H. van Olphen, Internal mutual flocculation in clay suspensions, JOURNAL OF COLLOID SCIENCE 19 (1964) 313-322.

[18] L. J. Michot, I. Bihannic, K. Porsch, S. Maddi, C. Baravian, J. Mougel, and P. Levitz, Phase Diagrams of Wyoming Na-Montmorillonite Clay. Influence of Particle Anisotropy, Langmuir 20 (2004) 10829_ 10837.

[19] L.J. Michot, E. Paineau, I. Bihannic, S. Maddi, Duval, J. F. L., C. Baravian, P. Davidson, P. Levitz, Isotropic/nematic and sol/gel transitions in aqueous suspensions of size selected nontronite NAu1, Clay Minerals 48 (2013) 663-685.

[20] E. Paineau, L.J. Michot, I. Bihannic, C. Baravian, Aqueous suspensions of natural swelling clay minerals. 2. Rheological characterization, Langmuir the ACS journal of surfaces and colloids 27 (2011) 7806-7819.

[21] S.J. Chipera and D.L. Bish, Baseline studies of the clay minerals society source clays powder X-Ray diffraction analyses, Clays and Clay Minerals 49.

[22] N. Cheng, X. Ma, X. Sheng, T. Wang, R. Wang, J. Jiao, L. Yu, Aggregation behavior of anionic surface active ionic liquids with double hydrocarbon chains in aqueous solution: Experimental and theoretical investigations, Colloids and Surfaces A: Physicochemical and Engineering Aspects 453 (2014) 53-61.

[23] A. EL Aferni, M. Guettari, T. Tajouri, Effect of polymer conformation on polymer-surfactant interaction in salt-free water, Colloid Polym Sci 294 (2016) 1097-1106.

[24] J. Thavorn, J.J. Hamon, B. Kitiyanan, A. Striolo, B.P. Grady, Competitive surfactant adsorption of AOT and Tween 20 on gold measured using a quartz crystal microbalance with dissipation, Langmuir the ACS journal of surfaces and colloids 30 (2014) 11031-11039.

[25] E.L. Michor, J.C. Berg, Micellization behavior of Aerosol OT in alcohol/water systems, Langmuir the ACS journal of surfaces and colloids 30 (2014) 12520-12524.

[26] Z.X. Li, J.R. Lu, G. Fragneto, R.K. Thomas, B.P. Binks, P. Fletcher, J. Penfold, Neutron reflectivity studies of Aerosol-OT monolayers adsorbed at the oil/water, air/water and hydrophobic solid/wate interfaces, Colloids and Surfaces A: Physicochemical and Engineering Aspects 135 (1998) 277-281.

[27] O. Ghosh, C.A. Miller, Liquid-crystalline and microemulsion phase behavior in alcohol-free Aerosol-OT/oil/brine systems, J. Phys. Chem. 91 (1987) 4528-4535.

[28] J. De Rozieres, Cinétique d'adsortpion des tensioactifs anioniques sur les argiles, 1985.

[29] S.H. Sutheimer, P.A. Maurice, Q. Zhou, Dissolution of well and poorly crystallized kaolinites; Al speciation and effects of surface characteristics, American Mineralogist 84 (1999) 620-628.

[30] J. Du, G. Morris, R.A. Pushkarova, R.S.C. Smart, Effect of surface structure of kaolinite on aggregation, settling rate, and bed density, Langmuir the ACS journal of surfaces and colloids 26 (2010) 13227-13235.

[31] M.S. Hassan, F. Villiéras, A. Razafitianamaharavo, L.J. Michot, Role of exchangeable cations on 
geometrical and energetic surface heterogeneity of kaolinites, Langmuir the ACS journal of surfaces and colloids 21 (2005) 12283-12289.

[32] Z. Li, L. Gallus, Surface configuration of sorbed hexadecyltrimethylammonium on kaolinite as indicated by surfactant and counterion sorption, cation desorption, and FTIR, Colloids and Surfaces A: Physicochemical and Engineering Aspects 264 (2005) 61-67.

[33] M.S. Hellsing, A.R. Rennie, A.V. Hughes, Effect of concentration and addition of ions on the adsorption of aerosol-OT to sapphire, Langmuir the ACS journal of surfaces and colloids 26 (2010) 14567-14573.

[34] W. Maier and A. Saupe, Eine einfache molekularstatistische Theorie der nematischen kristallinflüssigen Phase. Teil II, Z. Naturforsch. A. 15 (1960) 287

[35] W. Maier and A. Saupe, Eine einfache molekularstatistische Theorie der nematischen kristallinflüssigen Phase. Teil I., Z. Naturforsch. A. 14 (1959) 882.

[36] Maier W. and Saupe A., Z. Naturforsch. A. (1958) 564.

[37] E. Ferrage, F. Hubert, E. Tertre, A. Delville, L.J. Michot, P. Levitz, Modeling the arrangement of particles in natural swelling-clay porous media using three-dimensional packing of elliptic disks, Physical review. E, Statistical, nonlinear, and soft matter physics 91 (2015) 62210. 